\title{
ANALYTICAL MODELLING OF NORMAL AND OBLIQUE BALLISTIC IMPACT ON CERAMIC/METAL LIGHTWEIGHT ARMOURS
}

\author{
R. ZAERA* ${ }^{* \ddagger}$ and V. SÁNCHEZ-GÁLVEZ ${ }^{\dagger}$ \\ * Dpto. de Ingenieria. Escuela Politécnica Superior. Lniversidad Carlos III de Madrid. \\ C Butarque 15, 28911 Leganes, Madrid, Spain \\ 'Dpto. Ciencia de Materiales, E. T. S. Ingenieros de Caminos. Canales y Puertos. \\ Universidad Politécnica de Madrid. Ciudad Universitaria. 28040 Madrid. Spain
}

\begin{abstract}
Summary This paper presents a new analytical model developed to simulate ballistic impact of projectiles on ceramic/metal add-on armours. The model is based on Tate and Alekscevskiis equation for the projectile penetration into the ceramic tile, whilst the response of the metallic backing is modelled following the ideas of Woodward's and den Reijer's models. The result is a fully new analytical model that has been checked with datat of tesidual mass and residual velocity of real fire tests of medium caliber projectiles on ceramic/metal add-on armours. Agreement observed between experimental and analytical results confirmed the validity of the model. Therefore. the model developed can be a useful tool for optimisation of ceramic metal armour design.
\end{abstract}

\section{NOTATION}

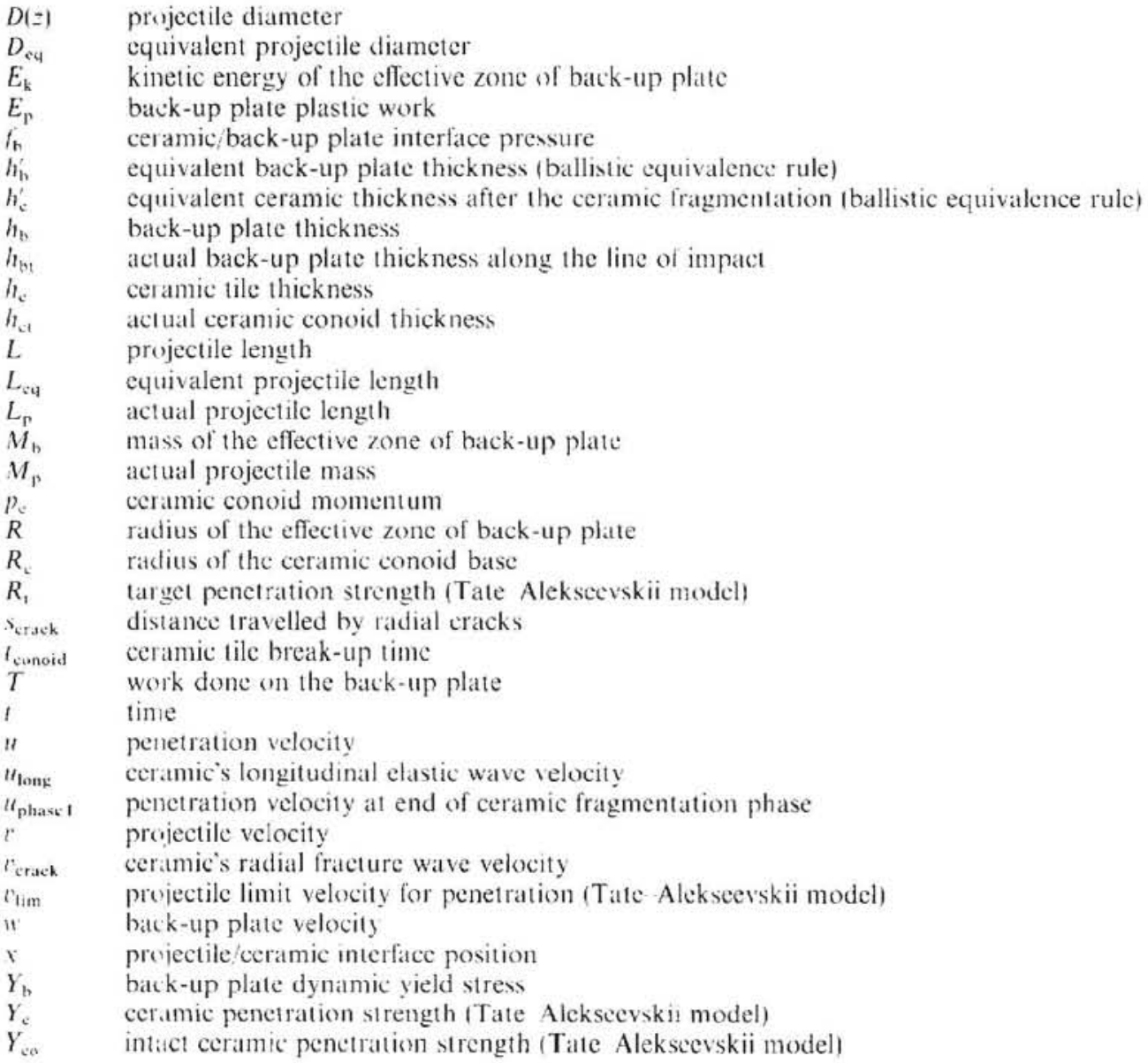

${ }^{4}$ Corresponding author. Tel: 341624 9920: lax: 341624 9430; c-mail: racra(aing.uc $3 m . e s$. 
$I_{p} \quad$ projectile strength (Tate-Alekseevskii model)

$x \quad$ ceramic conoid half-angle

i) central back-up plate deformation

(1) oblique impact angle (NATO)

is back-tup plate density

fos ceramic density

Ho projectile density

ti armour density

\section{INTRODUCTION}

Lightweight ceramic/metal armours designing is a quite complex task for which three different approaches might be followed: the empirical method, numerical simulation and analytical modelling. The empirical method is the most widely used, taken into account the difficulty of the analysis of such problems. It provides the highest accuracy but it has the disadvantage of being valid exclusively for the definite missile target system on hand. Semi-empirical techniques are usually developed in parallel, matching the experimental results, and can be used to extrapolate the results to other systems. Such techniques are not useful for a better understanding of the physical phenomena taking place during the penetration process. Numerical modelling derives a full solution of the penetration process by solving the whole set of differential equations of the mechanics of continuous media. The main advantage of this approach is the wider information provided which enables a better understanding of the process and it is quite valuable for an improved design of the armour. The accuracy of such codes is mainly dependent upon the definite constitutive equations used to represent the behaviour of each individual material. The relatively large CPU time required for a single simulation is a shortcoming for the utilisation of this approach as an armour design tool.

Finally, the third approach is the development of simple analytical models by assumıng some hypotheses which simplify the actual mechanisms of the penetration process. Material description is simplified by using simple equations and a few material parameters easily obtained by experimentation. The main advantage of this approach is that it provides the solution of a definite missile - target system in a few seconds with a personal computer, thus permitting the analysis of many different systems in a very short time. Disadvantages come from the lower accuracy of such models compared to full numerical simulation.

Up to now only three analytical models aiming to simulate the penetration process on ceramic metal armours have been fully developed [1-3]. Recently, another analytical model has been proposed by Walker and Anderson, but it is not yet fully developed [4]. All these models are one-dimensional and they analyse only the normal impact of a metallic projectile. In Florence's model, a global energy balance is proposed leading to the derivation of the ballistic speed limit. The more recent models by Woodward and den Rejer propose a set of equations governing the main physical mechanisms taking part during ihe penetration process. However, the deformation pattern of the target assumed by the three former models is identical. All the previously cited authors assume that a conoid of comminuted ceramic with a semiangle of about $65^{\circ}$ is developed which pushes forward a circular area of the metallic plate with dimensions equal or greater than that of the base of the ceramic conoid (Fig. 1).

With such hypothesis the force exerted by the projectile on the target is distributed over a circular area of the metal plate, which is much greater than the projectile section, so that hoth the inertia and the resisting force of the metal plate are quite high. Results achieved with these models are fairly accurate for the impact simulation of low caliber projectiles such as the 7.62 AP. However, when the previously mentioned models are used for the simulation of the impact behaviour of medium caliber projectiles, analytic results show a poor agreement with experimental data. as can be shown in Fig. 2, where residual velocities obtained by using Woodward's and den Reijer's models are compared to actual firing tests results performed by Empresa Nacional Santa Bárbara with $20 \mathrm{~mm}$ APIOS projectiles [5]. Therefore, these analytical models predict a higher resistance of the armour than what is actually observed. 


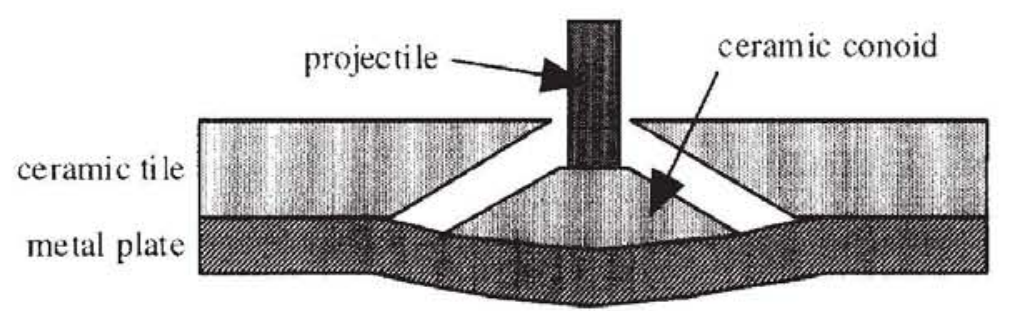

Fig. 1. Deformation pattern of the target in Florence. Woodward and den Rejjer models.

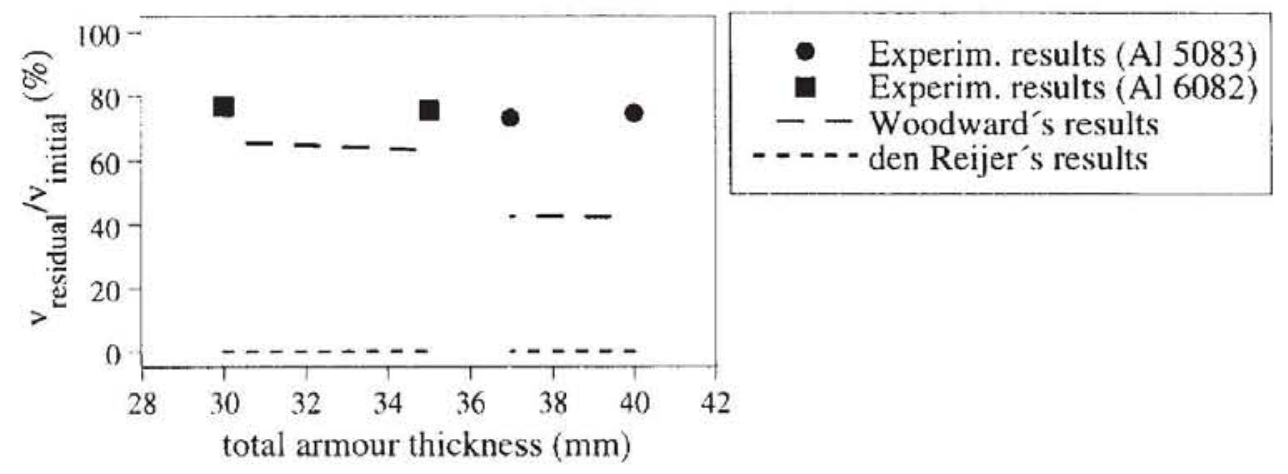

Fig. 2. Analytical and experimental results of impact residual velocities of 20 APDS projectiles onto alumina $99.5 \%$ aluminium alloy $(5083$ and 6082$)$ targets.

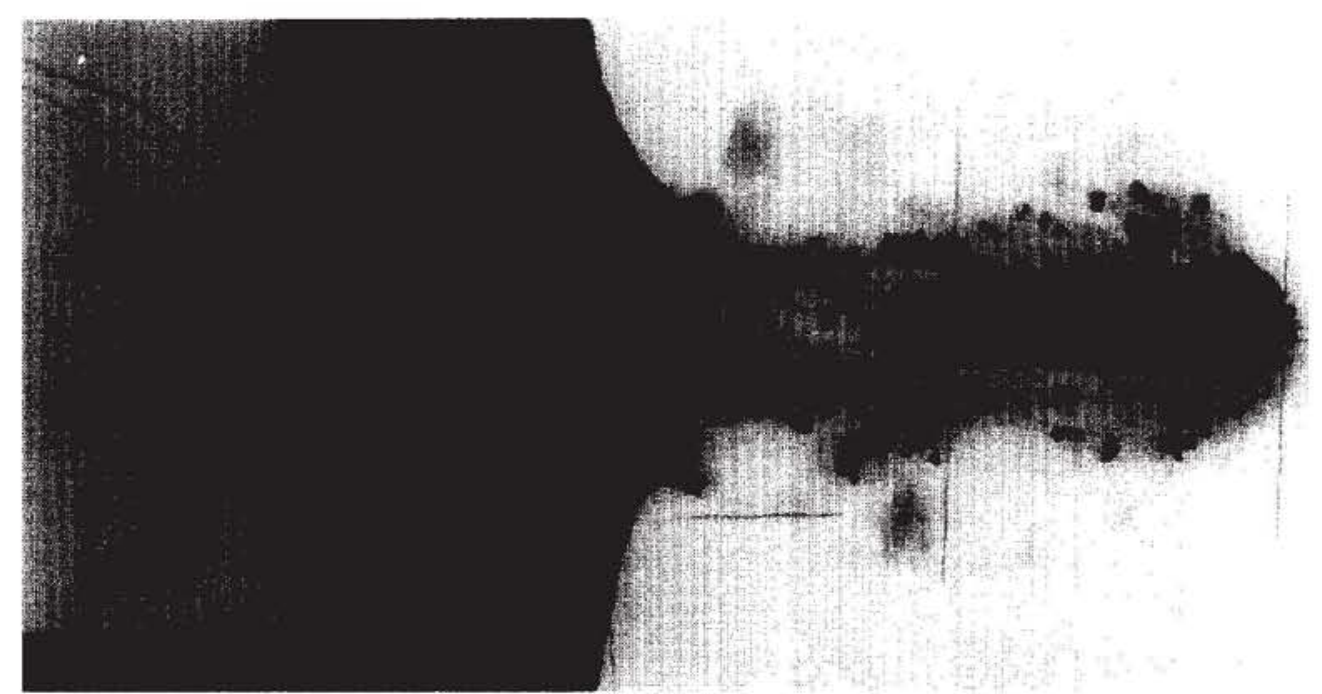

Fig, 3. X-ray shadowgraph of the impact of $25 \mathrm{~mm}$ APDS projectile on alumina aluminium target.

Medium caliber projectiles ( $20 \mathrm{~mm}$ APDS, $25 \mathrm{~mm}$ APDS, $30 \mathrm{~mm}$ APDS) include a core of high-density material such as tungsten and have higher muzzle velocities than those of low caliber projectiles. The penetration velocity is therefore much higher and the damage in the armour is more localised around the impact zone. X-ray shadowgraphs taken during the impact of $20 \mathrm{~mm}$ APDS projectiles on ceramic/metal targets [6] showed that the deformation of the metal plate is much more localised than it was assumed by former analytical models (Fig. 3).

\section{ANALYTICAL MODEL PROPOSED}

The model proposed was first developed to simulate normal impact of medium caliber projectiles on lightweight ceramic/metal armours [7] and its validity has been checked by 
comparing analytical predictions to experimental results of different ceramic/metal armours impacted by different low and medium caliber projectiles [8]. A more refined version of the model is presented here, aiming to simulate both normal and oblique impact of low and medium caliber projectiles.

\section{Modelling the projectile behatiour}

Mushrooming has been discarded in the analytical description of the projectile, thus only projectile erosion has been included in the model as well as rigid behaviour when erosion is negligible. As can be seen in the picture shown in Fig. 4, a $20 \mathrm{~mm}$ APDS projectile fired ¿gainst alumina/aluminium armour shows very little mushrooming. A similar behaviour has been reported by Mayseless et al. [9] for steel projectiles at different impact velocities.

For erosion description, the equation proposed by Tate [10] and Alekseevskii [11] has been chosen. It is similar to those used by Woodward and den Reijer, including a first term representing the material strength and a second one representing a hydrodynamic pressure. It has the advantage of being used by many researchers, who determined the values of the parameters of the equation fitting better the experimental bchaviour. Although Tate and Alekseevskii's model was first developed for metallic armours, many authors (Sternberg [12], Rosenberg et al. [13]. Hauver et al. [14], Hohler et al. [15], Ernst et al. [16]) have used it with advanced ceramics.

The equations of the model are

$$
\begin{gathered}
Y_{\mathrm{p}}+\frac{1}{2} \rho_{\mathrm{p}}(v-u)^{2}=R_{\mathrm{t}}+\frac{1}{2} \rho_{1} u^{2}, \\
\frac{\mathrm{d} L}{\mathrm{~d} t}=-(v-u) \\
\frac{\mathrm{d} v}{\mathrm{~d} t}=-\frac{Y_{\mathrm{p}}}{\rho_{\mathrm{p}} L}
\end{gathered}
$$

where $Y_{\mathrm{r}}$ is the dynamic strength of projectile material, $R_{\mathrm{t}}$ is the penetration strength of the target, $\rho_{\mathrm{p}}$ and $\rho_{\mathrm{t}}$ are the densities of projectile and target, respectively, $L$ the actual length of the projectile, $v$ its velocity and $u$ the penetration speed. Projectile behaviour is assumed to he rigid-perfectly plastic; thus the projectile is assumed to be undeformed except for a small zone near the projectile tip which is assumed to be plastic, and will then immediately be

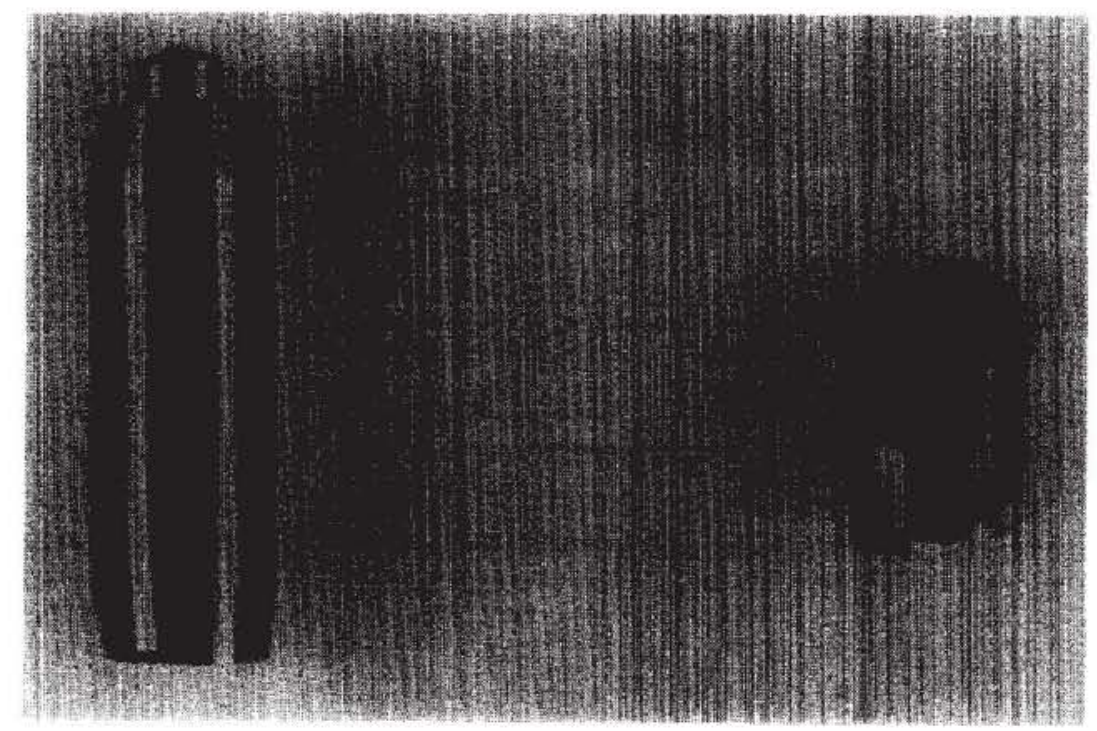

Fig. 4. A picture illustrating a 20 APIDS projectile before (left) and after (right) perforating a ceramic metal target. 
eroded. Such hypothesis is reasonable because the elastic energy stored in the projectile is negligible compared to the energy dissipated in plastic deformation and erosion.

When the projectile velocity has been sufficiently reduced, the pressure on its tip is not able to erode the projectile, therefore it will behave thereafter as a rigid body. Linear momentum equation in that case is

$$
\frac{\mathrm{d} v}{\mathrm{~d} t}=-\frac{R_{\mathrm{t}}+\frac{1}{2} \rho_{\mathrm{t}} v^{2}}{\rho_{\mathrm{p}} L} .
$$

Tate-Alekseevskii model considers an ideal projectile with perfect cylindrical shape, however actual projectiles usually have ogival or conical nose and a cylindrical body. Therefore, for noncylindrical projectiles, equivalent diameter and length have been determined, so that Tate-Alekseevskii model could be used with such projectiles. Equivalent diameter $D_{\text {eq }}$ has been computed by weighting each differential element of the projectile with its diameter, according to the following expression:

$$
D_{\mathrm{eq}}=\frac{\int_{0}^{L_{w^{\prime \prime}}} D^{3}(z) \mathrm{d} z}{\int_{0}^{L^{\prime \prime}} D^{2}(z) \mathrm{d} z},
$$

where $L_{\mathrm{r}}$ is the actual length of the projectile and $D(z)$ the diameter for position $z$. Equivalent length $L_{\mathrm{cq}}$ is finally determined by equating to the actual mass of the projectile:

$$
L_{\mathrm{cq}}=\frac{4 M_{\mathrm{p}}}{\pi D_{\mathrm{vq}}^{2} \rho_{\mathrm{p}}},
$$

where $M_{\mathrm{p}}$ is the projectile mass.

\section{Modelling the conoid of comminuted ceramic}

Fragmentation of the ceramic tile. One of the most important stages in the response of the ceramic tile under impact is the initial phase immediately after the contact with the projectile. During the first microseconds, a stress wave starts propagation from the impact surface, producing in the material a cracking front advancing in the impact direction. This front produces cracks well described in the literature [17] called circumferential and conical cracks. These cracks are generated by tensile stress waves propagating from the border of the impact area. Also in the vicinity of the projectile-ceramic interface, a volume (Mescall zone) may develop where the ceramic is pulverised because the contact pressure is higher than the compressive strength of the material. When the compressive wave reaches the rear surface of the tile, tensile circumferential stresses are generated which will propagate upwards.

Ceramic fragmentation is a continuous event taking place during the whole penetration process. However, several authors $[18,3]$ assume that fragmentation occurring during the first instants after impact is the most relevant on the decrease of ceramic strength. Crack generation, as described above. permits the motion of small fragments, making projectile penetration easier. Den Reijer proposes to build up a conoid of fragmented ceramic and to assume lower mechanical properties for the material inside the conoid than those of the intact ceramic. The time necessary to accomplish this stage is that required by different cracking fronts to go through the ceramic tile at an assumed constant speed.

Assuming that the conoid is fully fragmented when radial cracks generated at the rear surface of the tile reach the front face and also assuming that cracks are produced when compressive waves have travelled through the tile thickness, the time required to finish the first stage is

$$
t_{\text {conoid }}=\frac{h_{\mathrm{c}}}{u_{\text {long }}}+\frac{h_{\mathrm{c}}}{v_{\mathrm{crack}}},
$$

where $h_{\mathrm{e}}$ is the ceramic tile thickness, $u_{\text {long }}$ the speed of the longitudinal stress waves and $v_{\text {crack }}$ the speed of radial cracks propagation. In order to fit the values quoted by Wilkins 
[18] about the duration of the first phase, den Reijer assumes a value of $v_{\text {crack }}$ equal to one-fifth the value of $u_{\text {long. }}$. During this first stage, ceramic strength is the highest one since the tile is not yet fully cracked and the back-up metallic plate is still undeformed avoiding fragment motion. Therefore, den Reijer [3] assumes that the armour cannot be penetrated by the projectile during this stage.

For steel projectiles and impact velocities below about $800 \mathrm{~m} / \mathrm{s}$. experimental results of Wilkins and den Reijer show that the ceramic tile is not penetrated by the projectile during the first stage. but such conclusion cannot be sustained for tungsten projectiles and impact vclocities around $1200 \mathrm{~m} / \mathrm{s}$. Hydrocode simulations of two impacts have been performed, projectiles being similar to $20 \mathrm{~mm}$ APDS but with different materials and impact velocities. The armour is a $20 \mathrm{~mm}$ thick $99.5 \%$ purity alumina backed by $15 \mathrm{~mm}$ thick 5083 aluminium alloy. Ceramic material has been simulated by using the constitutive equation proposed by Cortes et al. [19]. For the first case analysed, a steel projectile at $800 \mathrm{~m} / \mathrm{s}$ wis considered, whilst in the second one, tungsten projectile impacting at $1250 \mathrm{~m} / \mathrm{s}$ was considered. As can be seen in Fig. 5, after $12 \mu$ s [approximate duration of the first stage according to Eqn (7)] penetration is negligible (less than $1 \mathrm{~mm}$ ) in the first case and it is quite noticeable in the second one (about $5 \mathrm{~mm}$ ).

Tate-Alekseevskii's equation leads to the same conclusion. The following equation

$$
l_{\text {lim }}=\sqrt{\frac{2\left(Y_{\mathrm{co}}-Y_{\mathrm{p}}\right)}{\rho_{\mathrm{p}}}}
$$

gives limit velocity for penetration into the armour, $Y_{\mathrm{co}}$ being the strength of intact ceramic. If appropriate values of parameters are introduced into Eqn (8) to simulate the impact processes analysed by Wilkins and den Reijer, for differences in ceramic and projectile strengths greater than $2.5 \mathrm{GPa}$ no penetration will be observed. Such condition is widely fulfilled according to observations made by Rosenberg et al. [13], Hauver et al. [14] and Hohler et al. [15]; in all cases strength measurements for different kinds of alumina provide average values over $5 \mathrm{GPa}$, and even greater values for the first stage of the impact process. However, when tungsten medium caliber projectiles are utilised, strength differences had to bc as high as $14 \mathrm{GPa}$ to avoid any initial penetration. Obviously, such condition is then very dilficult to fulfill and therefore we can conclude that for medium caliber projectiles (tungsten core and speeds over about $1000 \mathrm{~m} / \mathrm{s}$ ). a significant penetration may be observed before ceramic fragmentation.

Therefore, in the model proposed. even with intact ceramic. Tate-Alekseevskii's equiltions [Eqns (1) (3)] are used to determine the penetration speed $u$. When the value obtained is negative. we assume that the projectile is unable to penetrate the intact ceramic, the penctration speed $u$ is then taken as zero. If the penetration speed derived is positive, the time to finish the first stage must be changed, because the projectile tip will meet the cracking front before its arrival at the front surface. That means that projectile tip and

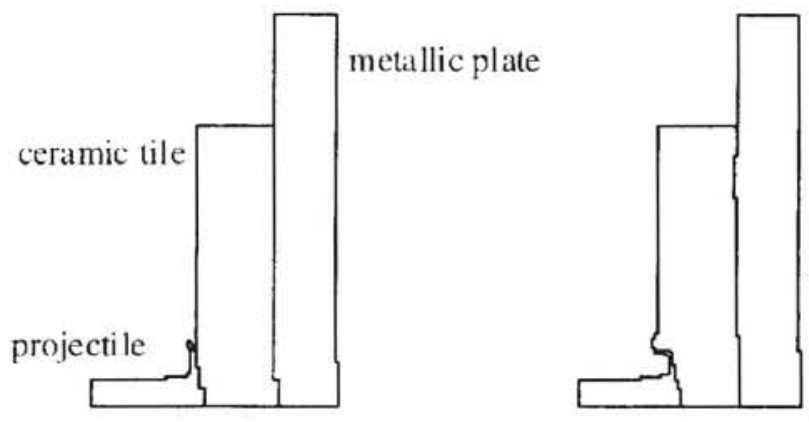

Fig. 5 . Impact of $12 \mathrm{~mm}$ diameter, $31.5 \mathrm{~mm}$ length projectile against a $20 \mathrm{~mm}$ alumina $99.5 .15 \mathrm{~mm}$ 5083 aluminium alloy target. Solid contours at $12 \mu \mathrm{s}$ after initial contact. Left: steel projectile at $800 \mathrm{~m} / \mathrm{s}$. Right: tungsten projectile at $1250 \mathrm{~m} / \mathrm{s}$. 


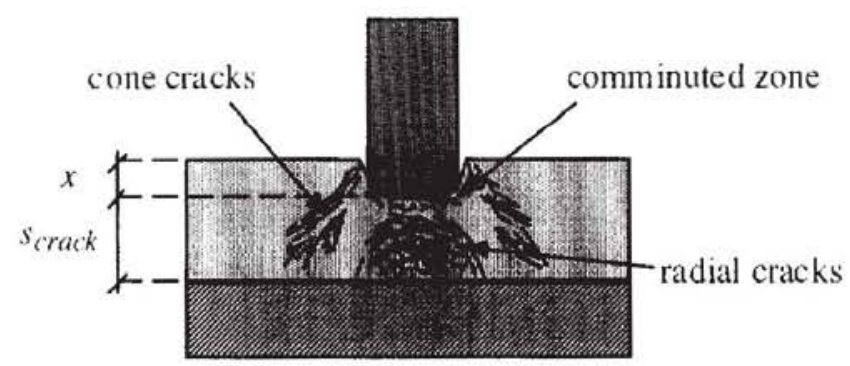

Fig. 6. Meeting of projectile tip and radial cracking front. End of fragmentation stage.

cracking front positions must be checked continuously along the first stage, ending it when both positions coincide (Fig. 6). Therefore. the condition for finishing stage one is given by

$$
x+s_{\text {crack }}=h_{i} .
$$

Behaviour of the conoid of fragmented ceramic. When the projectile tip meets the cracking front, the penetration proceeds into a volume of damaged ceramic whose mechanical properties have been reduced. The volume of fragmented ceramic has a conoid shape physically separated from the remaining intact ceramic tile by cracks. This conoid is accelerated in the impact direction and distributes the pressure on the backing metallic plate. Former analytical models assumed a constant conoid semiangle between 60 and 65 for the whole penetration process. However, conical crack formation is not limited to angles in that range; Wilson et al. [20] have obtained. among ceramic fragments collected after fire tests, conical cracks at angles much lower with respect to the impact axis, thus suggesting that the ccramic volume separated from the tile and impulsed by the projectile would be much smaller. Also hydrocode simulations of ballistic impact on ceramic/metal armours demonstrate that in many cases the effective ceramic conoid impulsed by the projectile has a semiangle much lower than those assumed by the former analytical models. Although experimental data of the ceramic conoid semiangle are not always available, some authors have detected dependencies on different impact parameters. For instance. Wilson et al. [20] have observed a marked relation with the ceramic thickness to metal thickness ratio, high values of such ratio leading to greater values of the ceramic conoid semiangle. On the other hand. the conoid volume is also dependent upon projectile velocity. for high impact velocities the damaged volume being more localised around the impact point. Following these observations, the conoid semiangle has to be a function of ceramic and metal plate thickness and impact velocity, although the values observed in numerical simulations for different materials, projectiles and thicknesses are usually between 20 and 30 .

Field of velocities in the ceramic conoid. The velocity field in the impact direction along the armour as obtained from numerical simulations show a distribution very far from a constant value: at the projectile/ceramic interlace, the ceramic particle velocity is equal to the penetration speed $u$. but it decreases very rapidly down to a slope fairly constant until the ceramic/metal interface. Finally, in the backing plate the speed is approximately constant. In the present model, such velocity field has been approximated by the distribution illustrated in Fig. 7.

With this assumption, the rate of linear momentum of the conoid can be expressed by Eqn $(10)$, where $f_{\mathrm{b}}$ is the pressure on the ceramic/metal interface, $R_{\mathrm{c}}$ the radius of the conoid base and $p_{c}$ its linear momentum (see Fig. 8):

$$
\frac{\mathrm{d} p_{\mathrm{c}}}{\mathrm{d} t}=Y_{\mathrm{c}} \pi \frac{D_{\mathrm{eq}}^{2}}{4}-f_{\mathrm{b}} \pi R_{\mathrm{c}}^{2} .
$$

In Fig. 8. $h_{\mathrm{c}}$ is the actual thickness of ceramic separating the projectile tip from the metal plate. Such parameter is decreasing with the penetration. In the same way the conoid base 


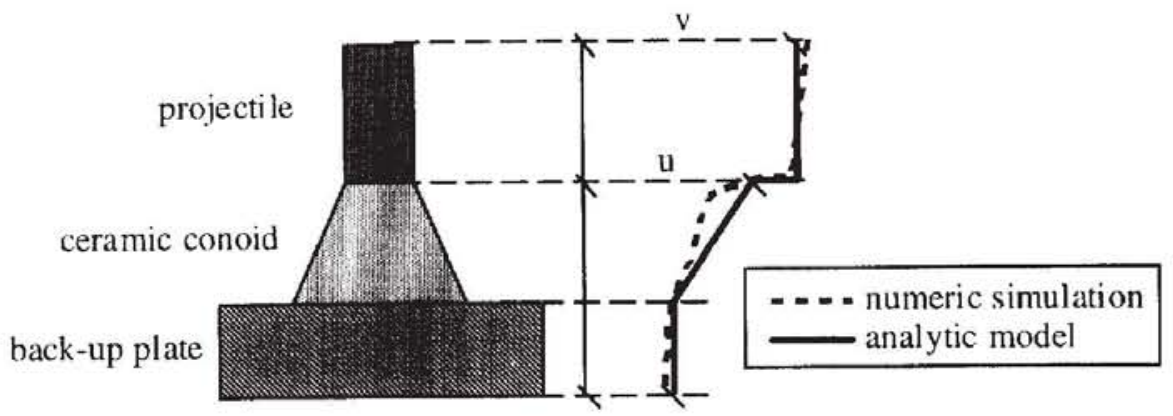

Fig. 7. Velocity field assumed for the target in the analytical model.

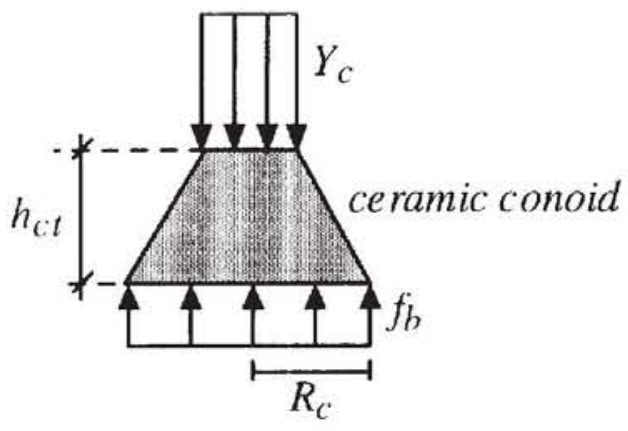

Fig. 8. Ceramic conoid desription.

radius $R_{\mathrm{c}}$ is also decreasing according to Eqn (11), where $\alpha$ is the conoid semiangle:

$$
R_{\mathrm{c}}=\frac{D_{\mathrm{eq}}}{2}+h_{\mathrm{cl}} \tan x \text {. }
$$

Linear momentum of the conoid $p_{\mathrm{c}}$ can be obtained taking into account that the assumed velocity distribution is linear being $u$ at projectile interface and $w$ at the metal-plate interface:

$$
p_{\mathrm{c}}=\pi \rho_{\mathrm{c}} h_{\mathrm{ct}}\left[u\left(\frac{D_{\mathrm{eq}}^{2}}{16}+\frac{R_{\mathrm{c}}^{2}}{12}+\frac{D_{\mathrm{eq}} R_{\mathrm{c}}}{12}\right)+w\left(\frac{D_{\mathrm{eq}}^{2}}{48}+\frac{R_{\mathrm{c}}^{2}}{4}+\frac{D_{\mathrm{eq}} R_{\mathrm{c}}}{12}\right)\right] .
$$

The rate of change of the linear momentum can then be derived:

$$
\frac{\mathrm{d} p_{\mathrm{c}}}{\mathrm{d} t}=\frac{\partial p_{\mathrm{c}}}{\partial h_{\mathrm{e} t}} \frac{\mathrm{d} h_{\mathrm{e} t}}{\mathrm{~d} t}+\frac{\partial p_{\mathrm{c}}}{\partial R_{\mathrm{c}}} \frac{\mathrm{d} R_{\mathrm{c}}}{\mathrm{d} t}+\frac{\partial p_{\mathrm{c}}}{\partial u} \frac{\mathrm{d} u}{\mathrm{~d} t}+\frac{\partial p_{\mathrm{c}}}{\partial w} \frac{\mathrm{d} w}{\mathrm{~d} t} .
$$

Tate-Alekseevskii's equation must now be changed by introducing the velocity $w$, in the second term of the right-hand side. because the projectile is penetrating a nonstationary medium:

$$
Y_{\mathrm{p}}+\frac{1}{2} \rho_{\mathrm{p}}(l-u)^{2}=R_{\mathrm{t}}+\frac{1}{2} \rho_{\mathrm{t}}(u-w)^{2} .
$$

According to Tate-Alekseevskii's equation, $R_{\mathrm{t}}$ is the penetration strength of the material facing the projectile. Therefore, $R_{\mathrm{t}}$ is equal to the ceramic penetration strength $Y_{\mathrm{c}}$ when the projectile is travelling through the ceramic tile, and is equal to the back-up plate dynamic yield stress $Y_{\mathrm{b}}$, when it is penetrating the metal plate.

Ceramic penetration strength $Y_{\mathrm{c}}$. Ceramic penetration strength is dramatically lowered after fragmentation. When the ceramic tile loses its integrity, it becomes a granular medium with reduced mechanical properties. Wilkins [18] proposed the utilisation of two different sets of mechanical properties, the first one for intact ceramic and the second one for damaged material. In the present model. Wilkins' proposal has been followed by assuming 
a constant value $Y_{\text {co }}$ for the ceramic strength during fragmentation and a lower value when conoid formation has been completed. The resistance to penetration of ceramic fragments is highly dependent on ceramic confinement. The metal plate being deformed and some particles being expelled from the crater. more room for the fragments motion is available and thus the penetration resistance decreases [3]. Following these ideas, the expression for fragmented ceramic strength assumed in the model is

$$
Y_{\mathrm{c}}=Y_{\mathrm{co}}\left(\frac{u-w}{u_{\text {phase } 1}}\right)^{2},
$$

where $u_{\text {phase } 1}$ is the penetration velocity at the end of fragmentation stage, when the ceramic strength decrease starts.

\section{Modelling the behaviour of the metal plate}

Effectire zone and velocity field assumed. In analytical modelling of impact problems the analysis of the target is usually restricted to an effective zone, which is the most relevant on the impact process and assuming simple velocity fields on those areas of the effective zone for which the speed gradients are not very important [21]. In the ceramic, being a granular aterial, speed change between the projectile interface and the metal plate is high. therefore a nonuniform speed distribution has been adopted. However, numerical simulations show very low velocity gradients in the metal plate in the impact direction. In the radial direction. the velocity gradients are higher especially for the last stages of the penetration when the projectile meets the metal plate and the process is concentrated in a narrow zone close to the impact axis. Anyway, the speed change in the metal plate is much lower than that in the ceramic conoid, thus a constant value $w$ can be adopted for the whole effective zone.

Derivation of the magnitude of the effective zone has been carried out based on kinematic criteria. As can be seen in Fig. 9, velocity in the impact direction as obtained from numerical simulations, shows a marked reduction at a definite distance from impact axis. Such distance is approximately equal to the conoid base radius $R_{\mathrm{c}}$ at the beginning of the penetration and it is kept fairly constant for the whole process. Summarising, although the conoid base radius $R_{\mathrm{c}}$ is decreasing with the resisting ceramic thickness, the effective zone radius in the metal plate $R$ is assumed to be constant and equal to the value of $R_{\mathrm{c}}$ at the beginning of the penetration:

$$
R=\frac{D_{\mathrm{cu}}}{2}+h_{\mathrm{c}} \tan \alpha .
$$

Energy description of the problem: equation of motion of the metallic plate. Analytical modelling of metal plates subjected to impulsive loads can be performed by expressions based on energy balance or by equations of momentum rate of change. In the first

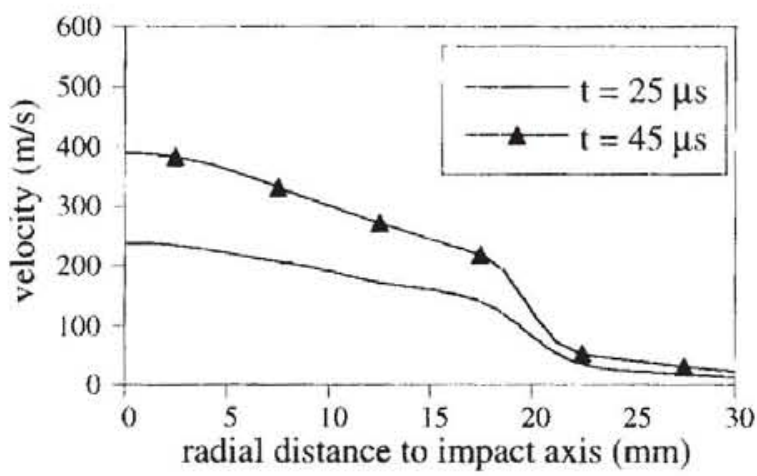

Fig. 9 Velocity in the impact direction versus distance to impact axis for two different times. Projectile: 20 APDS at $1250 \mathrm{~m}$ s. Target: $20 \mathrm{~mm}$ alumina, $15 \mathrm{~mm} 5083$ aluminium alloy. 
approach, the problem is usually formulated by uncoupling time and position in the equation of the deformed plate, so that the displacement of any point is given by the product of a shape function dependent on the position of the point by the displacement of the plate axis. From this equation and the strain field derived from it, the energy dissipated $E_{\mathrm{r}}$ by plastic deformation can be determined. In the second approach, a distribution of stresses on the effective zone border is assumed. usually the yield stress being assumed as the stress acting on the border, to simplify the model.

In the present model, the first approach has been followed, since it provides enough accuracy. Work dissipated by plastic deformation is obtained by Eqn (17) as proposed by Woodward et al. [22], including plastic work done in stretching and in bending of the plate:

$$
E_{\mathrm{r}}=\pi h_{\mathrm{b}} \delta Y_{\mathrm{b}}\left(\frac{2}{3} h_{\mathrm{b}}+\frac{1}{2} \delta\right) \text {. }
$$

where $h_{\mathrm{b}}$ is the plate thickness, $\delta$ is the central plate deformation and $Y_{\mathrm{b}}$ its dynamic yield stress. The plastic work rate of change is thus:

$$
\frac{\mathrm{d} E_{\mathrm{p}}}{\mathrm{d} t}=\pi h_{\mathrm{b}} Y_{\mathrm{h}} w\left(\frac{2}{3} h_{\mathrm{b}}+\delta\right) .
$$

On the other hand, the rate of change of the work done by forces acting on the interface ceramic/metal is (see Fig. 10)

$$
\frac{\mathrm{d} T}{\mathrm{~d} t}=\pi f_{\mathrm{b}} R_{\mathrm{c}}^{2} w .
$$

Finally, for the effective zone defined above, the kinetic energy rate of change can he expressed as

$$
\frac{\mathrm{d} E_{\mathrm{k}}}{\mathrm{d} t}=\pi R^{2} h_{\mathrm{b}} \rho_{\mathrm{b}} w \frac{\mathrm{d} w}{\mathrm{~d} t},
$$

and the energy balance equation is

$$
f_{\mathrm{b}} R_{\mathrm{c}}^{2}=h_{\mathrm{h}} Y_{\mathrm{h}}\left(\frac{2}{3} h_{\mathrm{b}}+\delta\right)+R^{2} h_{\mathrm{b}} \rho_{\mathrm{b}} \frac{\mathrm{d} w}{\mathrm{~d} t} .
$$

Penetration of projectile into the metal plate. For high speed impacts the projectile may fully erode the ceramic tile and meet the front face of back-up plate. In that case, the projectile continues penetration into the metal plate until complete perforation or projectile arrest. In the present model, the actual behaviour is simulated allowing a different speed for projectile and metal plate and utilising Tate-Alekseevskii's erosion equation, which leads to the following expression for the projectile motion:

$$
\frac{\mathrm{d} t}{\mathrm{~d} t}=-\frac{Y_{\mathrm{b}}+\frac{1}{2} \rho_{\mathrm{b}}(v-w)^{2}}{\rho_{\mathrm{p}} L} .
$$

With respect to the metal plate. the equation of motion (23) is derived similarly to derivation of Eqn (21):

$$
\frac{\mathrm{d} w}{\mathrm{~d} t}=\frac{\frac{1}{4} \pi Y_{\mathrm{b}} D_{\mathrm{cu}}^{2} / 4-\pi h_{\mathrm{b}} Y_{\mathrm{b}}\left(\frac{2}{3} h_{\mathrm{b}}+\delta\right)}{M_{\mathrm{b}}}
$$

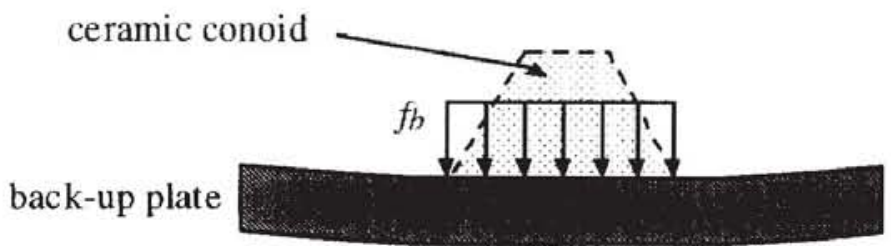

Fig. 10. Force acting on the metal plate. 


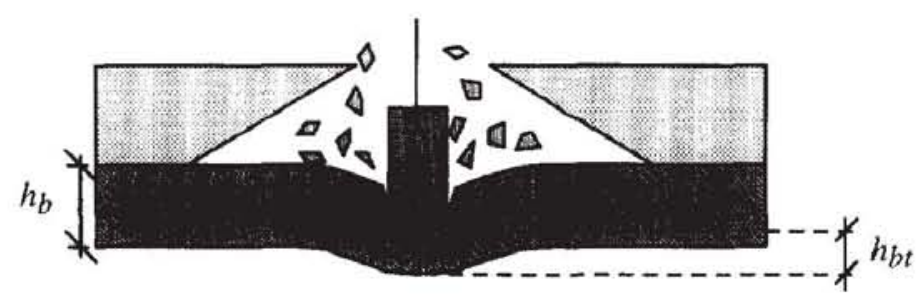

Fig. 11. Fully erosion of ceramic and contact of projectile with the metal plate.

where $M_{\mathrm{b}}$ is the mass of the effective zone given by

$$
M_{\mathrm{b}}=\pi \rho_{\mathrm{b}}\left(R^{2} h_{\mathrm{b}}-\frac{D_{\mathrm{cu}}^{2}}{4}\left(h_{\mathrm{b}}-h_{\mathrm{b}}\right)\right),
$$

with $h_{\mathrm{b}}$ being the actual central thickness of the plate as can be seen in Fig. 11.

In ceramic-metal armours, the main function of the back-up metal plate is ceramic fragment retention, its contribution to projectile deceleration when the projectile is in contact with the plate being much less important. Therefore. the effect of metal resistance decrease due to thickness reduction has been neglected.

\section{Failure criterion}

Two different failure criteria have been proposed to define full perforation of the armour. The first one is typical of impact at speeds much higher than the ballistic limit. In such cases, the projectile erodes fully the ceramic tile. meets the back-up metal plate and finally perforates it without producing important bending of the metal plate. Perforation criterion is then fulfilled when central metal plate thickness is reduced to zero, which means according to the proposed model.

$$
h_{\mathrm{h}}=0 \text {. }
$$

The second criterion is typical for lower impact speeds for which the metal plate shows important bending deformations. As can be seen by numerical simulations, when projectile speed $t$ is approximating metal speed $w$, the armour is defeated even though a full perforation of the ceramic tile or the metal plate is not yet achieved. Therefore, a kinematic failure criterion has been chosen, assuming armour defeat when

$$
r=w \text {. }
$$

\section{MODELLING OBLIQUE IMPACT}

\section{Ballistic equivalence rule (BER)}

The main advantage of analytical models as armour design tools is its computation speed. For this reason, all of them consider only uniaxial problems or two-dimensional problems with axial symmetry. Oblique impact is obviously a three-dimensional problem; consequently, analytical modelling to maintain its simplicity usually convert it into an equivalent normal impact by means of some hallistic equivalence rule (BER). Such rules are simply a clever modification of those parameters controlling the penetration process. By the application of BER, a new equivalent normal impact problem is defined, which can be solved by the analytical model. Finally. BER is applied again to convert the solutions required of the actual oblique problem.

Usually, modification is done exclusively on the geometric parameters defining the problem. For instance, in the case of metallic targets a simple rule often used is the transformation of the actual oblique impact on a plate of thickness $h_{t}$ into an equivalent normal impact on a thicker plate the equivalent thickness $h_{t}^{\prime}$ being given by

$$
h_{t}=\frac{h_{t}}{\cos \theta} .
$$


where $\theta$ is the impact angle (the angle between the impact direction and the normal to the target surface). In the present model, a geometric BER has been adopted, following basically Eqn (27) both for the ceramic tile and the back-up metal plate. Residual velocity and residual mass of the projectile after the perforation of the equivalent normal impact target are adopted without any additional change as the prediction for the actual oblique impact problem. Using such a simple equivalence rule, a good agreement with experimental results is obtained keeping the one-dimensional modelling developed for normal impact simulition.

However, application of such simple BER for the whole penetration process would provide projectile residual masses lower than experimental values. The explanation of such discrepancy is the duration of the fragmentation stage which is shorter in actual cases than that assumed by the equivalent normal impact problem. since the actual ceramic tile is thinner than the equivalent one. To solve this difficulty, BER is not applied for the fritgmentation stage, for which actual projectile trajectory is simulated. Although oblique impact is three-dimensional, the stress field produced during the first microseconds keeps the axial symmetry observed in normal impact because stress wave propagation is radial from the impact point. Ceramic fragmentation process is thus very similar to that occuring in normal impact cases, conical cracks propagating from the rear face of the tile. In Fig. 12, the radial cracks pattern produced in an oblique impact case can be observed, showing axial symmetry.

The condition for finishing the fragmentation stage is the same as for normal impact. It is finished when the projectile tip meets the radial cracking front propagating from the rear face of the tile. Therefore, Eqn (9) is substiluted by (see Fig. 13)

$$
x \cos \theta+s_{\text {crack }}=h_{\mathrm{c}} .
$$

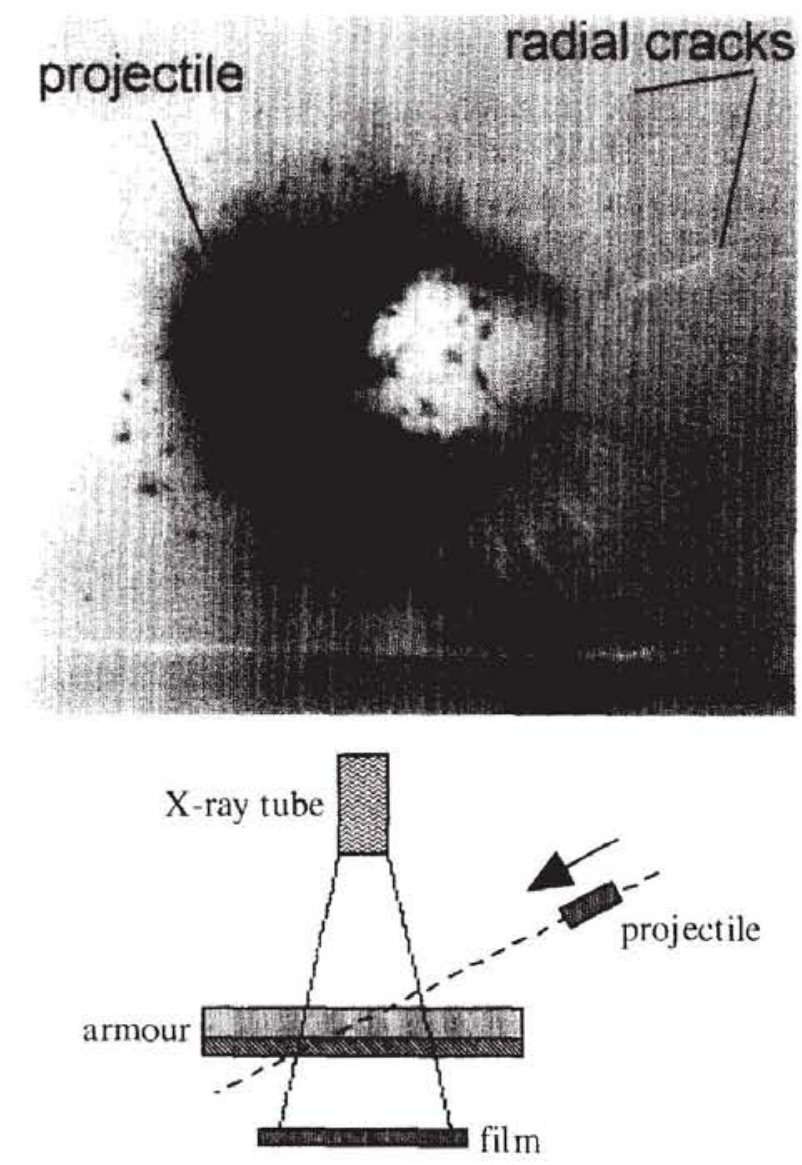

Fig. 12. Left: front image of oblique of $20 \mathrm{APDS}$ projectile against alumina/aluminum target. Right: sketch of experimental set-up (Empresa Nacional Santa Bárbarat. 


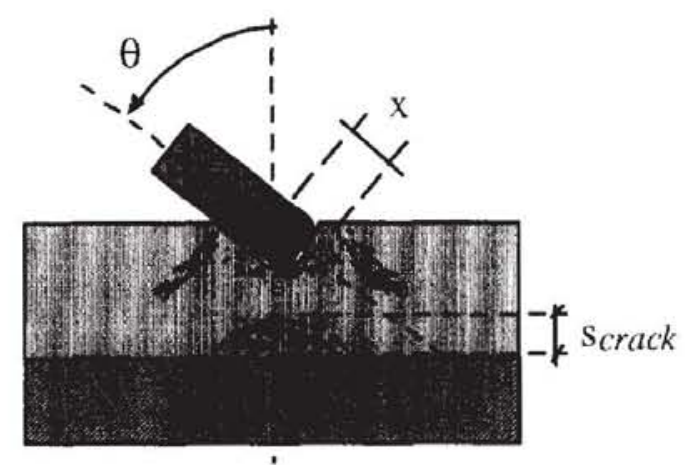

Fig. 13. Oblique penetration of projectile during ceramic fragmentation stage.

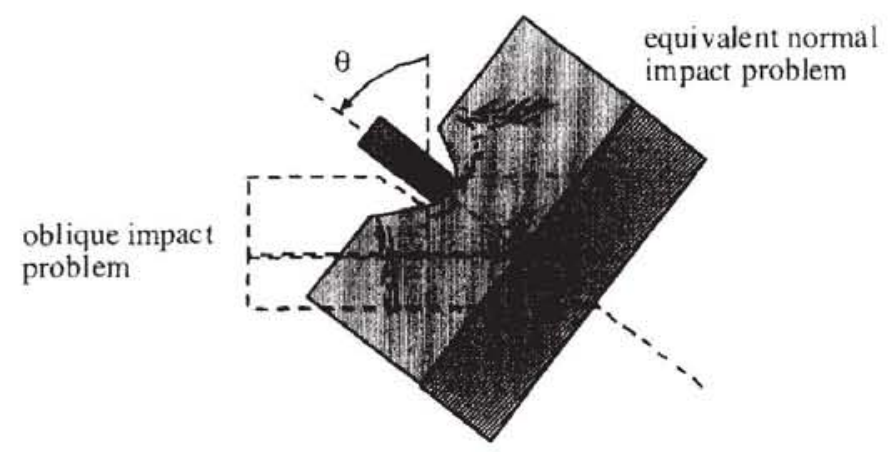

Fig. 14. Conversion to normal impact by the Ballistic Equivalence Rule.

The simple BER is applied thereafter utilising Eqns (29) and (30) with equivalent thicknesses $h_{\mathrm{c}}^{\prime}$ and $h_{\mathrm{b}}^{\prime}$ (see Fig. 14):

$$
\begin{gathered}
h_{\mathrm{c}}^{\prime}=\frac{h_{\mathrm{c}}}{\cos \theta}-x, \\
h_{\mathrm{b}}^{\prime}=\frac{h_{\mathrm{b}}}{\cos \theta} .
\end{gathered}
$$

\section{RESULTS OF THE PROPOSED ANALYTICAL MODEL}

In order to perform the simulations, the proposed $Y_{\mathrm{p}}$ and $Y_{\mathrm{co}}$ values for the projectiles and ceramics have been estimated from the ones given in Refs $[13,15]$.

\section{Small caliber projectiles}

For validating the model for small caliber projectiles, experimental results obtained by Wilkins [18] have been used. In Fig. 15, experimental ballistic limits of 7.62 AP projectiles impacting on AD 85 ceramic tiles backed by 6061 aluminium alloy plates have been plotted as well as the results of analytical simulations using Woodward's model and the proposed model.

Results of the proposed model are in good agreement with experimental results both for thin backing plates and for thick ones, maximum deviation being less than $10 \%$.

\section{Medium caliber projectiles}

For medium caliber projectiles (20 mm APDS, $25 \mathrm{~mm}$ APDS, $30 \mathrm{~mm}$ APDS), validation of the model has been carried out using a great number of experimental results obtained by 


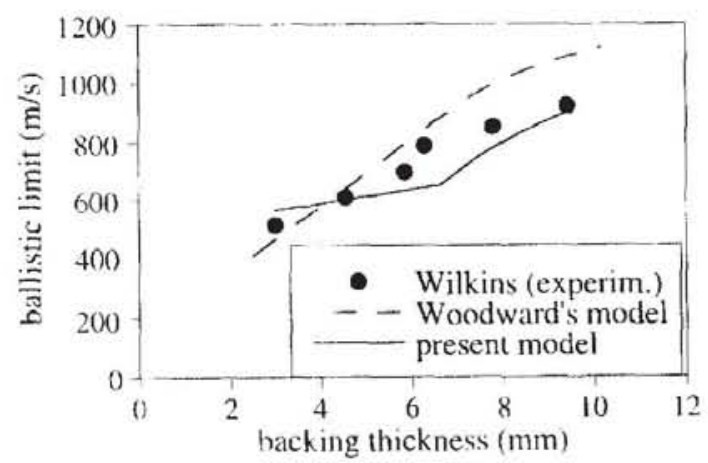

Fig. 15. Comparison of experimental ballistic limits of Wilkins with analytical results of Woodward's model and proposed model $7.85 \mathrm{~mm}$ AD85 + 6061-T6 aluminium alloy).

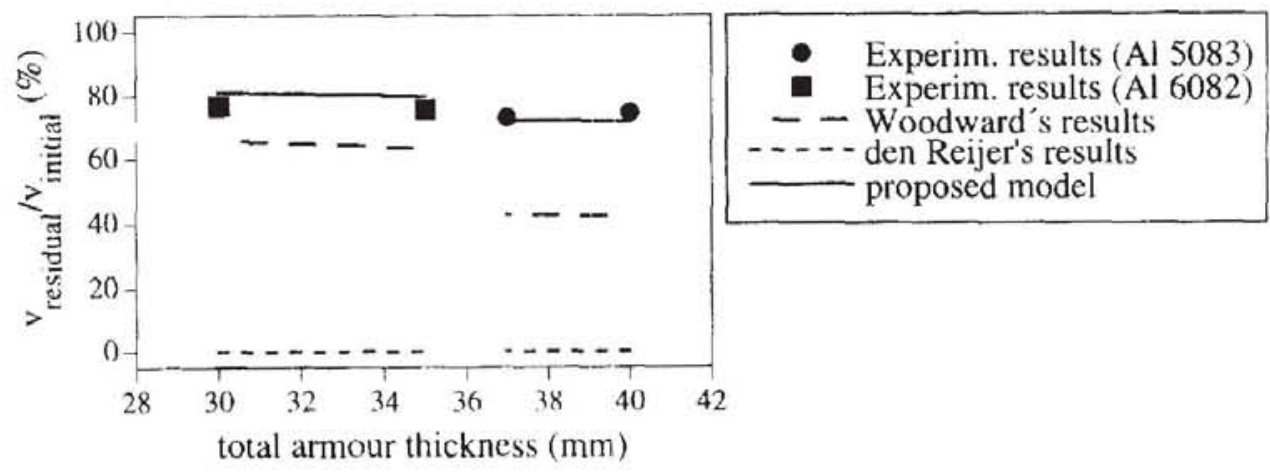

Fig. 16. Analytical and experimental results of impact residual velocities of 20 APDS projectiles onto alumina $99.5 \%$ altuminium alloy 15083 and 6082 ) targets.

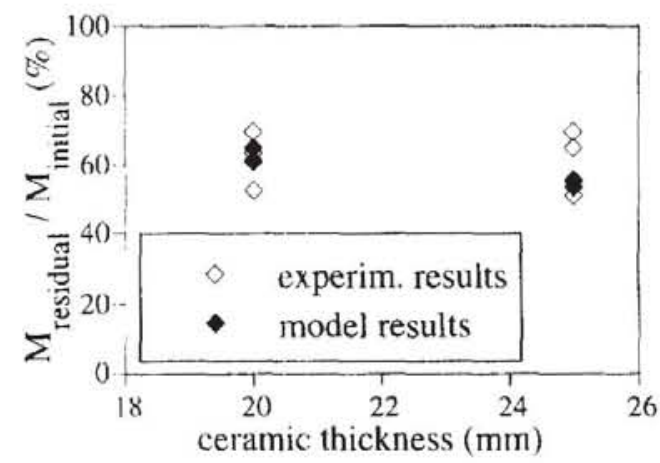

Fig. 17. Analytical and experimental results of projectile residual velocitics of 20 APDS projectiles onto alumina $99.5^{\circ} \%$ aluminium alloy $(5083$ and 6082$)$.

Empresa Nacional Santa Bárbara. Due to classification of fire test results, only a feu of them have been included in this paper, but it is enough to demonstrate the degree of improvement achieved as compared to existing analytical models. For instance. Fig. 16 illustrates the same results shown in Fig. 2 but the results of proposed model are now included showing a better agreement to experimental results than that achieved with Woodward's and den Reijer's model.

With respect to projectile residual masses, predictions of the model show also good agreement to actual masses of projectiles after impact as can be seen in Fig. 17 (experimental data from Peskes et al. [5]). In Fig. 18, additional comparisons are carried out between experimental data and analytical results for different targets and projectiles.

A good agreement between residual velocities experimental results and predictions of the present model is observed also with $30 \mathrm{~mm}$ APDS projectiles. Residual lengths predictions 

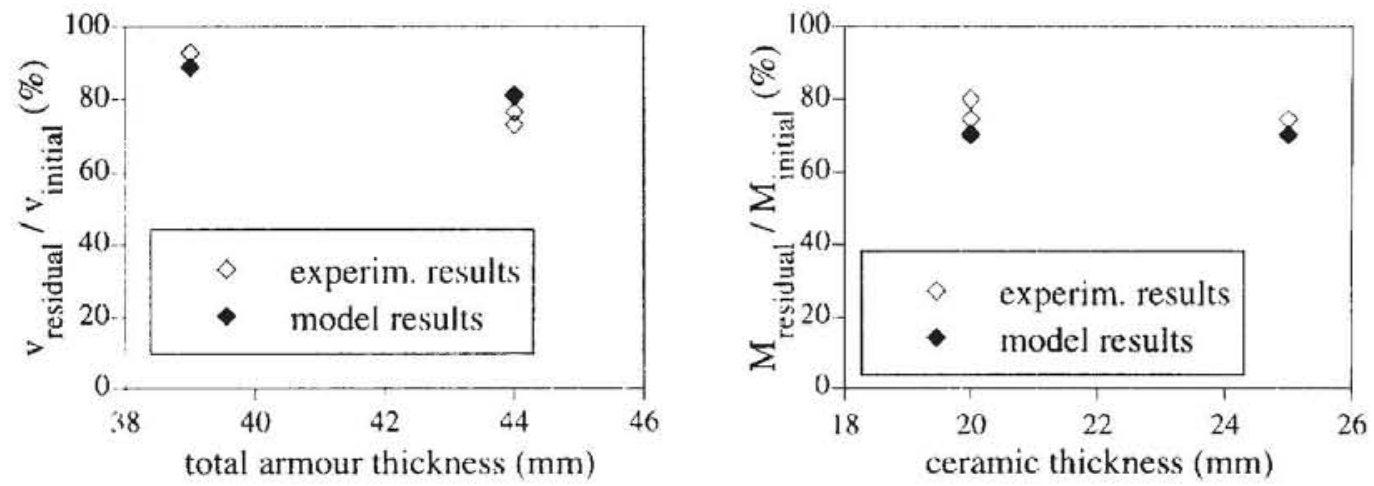

Fig. 1x. Analytical and experimental results of projectile residual velocities and residual masses of 25 APDS projectiles onto alumina $99.5 \%$ aluminium alloy 6082 .
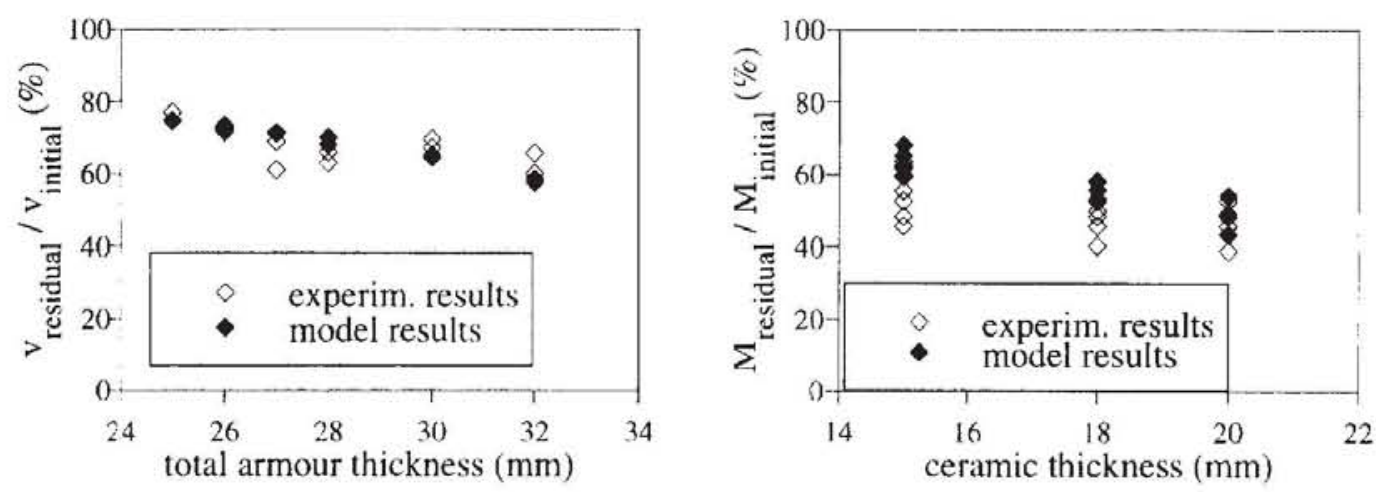

Fig. 19. Analytical and experimental results of projectile residual velocities and residuai masses of 20 APDS projectiles onto alumina $99.5^{\circ} \%$ aluminium alloy 6082: Impact angle: 50 VATO

are quite accurate too for $20 \mathrm{~mm}$ APDS and $25 \mathrm{~mm}$ APDS projectiles, although for $30 \mathrm{~mm}$ APDS projectiles a lower erosion is predicted than that observed experimentally. Probably. this discrepancy is due to the hypothesis of the model which assumes cylindrical projectiles, whilst the actual $30 \mathrm{~mm}$ APDS projectile includes a cylindrical hole in its back that reduces its strength.

\section{Oblique impact}

Figure 19 illustrates residual velocities and residual masses of $20 \mathrm{~mm}$ APDS projectiles after perforation of ceramic/metal targets at 50 NATO impact angles. In actual firing tests. the impact velocity is not constant; there is a little scatter in actual impact velocities. This is the reason of the scatter observed in analytical results plotted in the figures. The figures show a good agreement between experimental data published by Peskes et al. [5] and analytical results of the model proposed.

\section{CONCLUSIONS}

A new analytical model for simulating ballistic impact on ceramic/metal armours is presented. The model permits a very fast computation of ballistic limits, residual velocities and residual masses, being a very useful designing tool for ceramic/metal armours. The model has been utilised both for small caliber projectiles and medium caliber projectiles, perforating different ceramic/metal targets at different impact angles, analytical results showing a good agreement to experimental data. 
Acknowledgements The authors are indebted to the Research and Development Department of Empresat Vacional Santa Bárbara (Spain) for the performance of the ballistic tests and obtaining the X-ray shadowgraphs. This work is a part of the EUCLID RTP 3.2 projec:, supported by the Ministries of Defence of Spain, Denmark. Holland and Italy. The above project is being developed with the collaboration of several companies and reseitreh centres of the aforementioned countries.

\section{REFERENCES}

1. A. L. Florence, Interaction of projectiles and composite armour. Part II. Stanford Research Institute. Menlo Park. California, U.S.A., 1969.

2. R. L. Woodward, A simple one-dimensional approach to modelling ceramic composite armour defeat. $1 \mathrm{~m} . \mathrm{J}$ Impact Engng., 9(4). 455-474 (1990).

3. P. C. den Reijer. Impact on Ceramic Faced Armours. Ph.D. Thesis, Delft University of Technology (1991).

4. J. D. Walker and C. E. Anderson Jr., An analytical model for ceramic-faced light armors. Proc. on the $16 h$ Int Symp on Bullistics, San Francisco (1996).

5. G. J. J. M. Peskes. C. Briales. M. Gualco, M. Pellegri and S. Madsen, Optimisation of light weight amour. Proc 16 th Int. Symp. an Ballistics, San Francisco. U.S.A. (1996).

6. C. Briales. R. Cortés. R. Záera. M. A. Martinez and V. Sánchez-Gálvez. An experimental and numerical study on the impact of ballistic projectiles onto ceramic/metal armours. Prec. on the $15 \mathrm{th} \mathrm{Int}$. Simp. on Ballistics. Jerusalem. Israel $\{1995\}$.

7. V. Sánchez-Gálvez, R. Zaera. I. S. Chocrón. M. A. Martínez and R. Cortés, Modeling of composite armour panels subjected to ballistic impact. Proc. Lightweight Armour System Simp., Royal Military College of Science, Cranticld (1995).

8. R. Zaera and V. Sánchez-Gálvez, Analytical model of ballistic impact on ceramic metal lightweight armours. Proc 16h Int. Symp. on Ballistics, San Francisco (1996).

9. M. Mayseless, W. Goldsmith. S. P. Virostck, S. A. Finnegan, Impact on Ceramic Targets. J. Appl. Mech. 54. 373.378 (1987).

10. A. Tate, A theory for the deceleration of long rods after impact. J. Mech. Phys. Solids. 15, 387 399 1967).

11. V. P. Aleksecvskii. Penetration of a rod into a target at high velocity, in Combustion. Explosion and Shoch Wates. Vol. 2. Faraday Press. Neu York. UISA (1966).

12. J. Sternberg. Material poperties determining the esistance of ceramics to high velocity penetration, $J$. A ppt Phis.. 65, $34173424(1989)$.

13. Z. Rosenberg and $\mathbf{J}$. Tsaliah. Applying Tate's model for the interaction of long rod projectiles with ceramic largets, Int. J. Impact Engng 9(2). 247-251 (1990:

14. G. E. Hauver. P. H. Netherwood. R. F. Benck. W. A. Gooch, W. J. Perciballi and M. S. Burkins, Variation of target resistence during long rod penetration into ceramics. Proc 13th Imt. Symp. om Ballistics, Stockholm. Sweden (1992).

15. V. Hohler. A. J. Stilp and K. Weber. Hypervelocity penctration of tungsten sinter-alloy rods into alumina Int. J. Impact Engmy 17, 409418,1995$).$

16. H. J. Ernst. K. Hoog and V. Wiesner. Continusus measurements of the penetration depth in completely confined ceramic targets. Proc 15th Int. Symp. on Ballistics, Jerusalem, Israel (1995).

17. D. A. Shockey. A. H. Marchand, S. R. Skaggs, G. E. Cort, M. W. Burkett and R. Parker, Fature phenomenology of confined ceramic targets and impacting rods. Im. J. Impact Enymul. 9(3). 263275 (1990).

18. M. L. Wilkins. Mechanics of penetration and perforation. Int. J. Impact Engng. 16, $793 \quad 807$ (1978).

19. R. Cortes. C. Navarro, M. A. Martines, J. Rodriguez and V. Sánchez-Galvez, Numerical modelling of normal impact on ceramic composite armours, Int. J. Impact Engnu 12.639.651 (1992).

20. D. Wilson. J. G. Hetherington. Analysis of ballistic impact on ceramic faced armour using high speed photography, Prece Lightweight Armour Systcm Simp. Royal Military College of Science. Cranfield (1995).

21. J. A. Zukas, T. Nicholas, H. F. Swifı, L. B. Greszczuk, D. R. Curran. In Impact Dynamics. Wiley, New York. USA (1992)

2. R. L. Woodward, R. G. O'Donnell. B. J. Baxter, B. Nicol and S. D. Pattic. Energy absorption in the failure of ceramic composite armours, Mater. Form, 13, 174 181 (1989). 